\title{
INEQUALITIES VIA POWER SERIES AND CAUCHY-SCHWARZ INEQUALITY
}

\author{
MoOnja JEOng
}

\begin{abstract}
In this note we study Nesbitt's Inequality and modify it to make several inequalities. We prove some inequalities by using power series and Cauchy-Schwarz Inequality.
\end{abstract}

\section{INTRODUCTION}

Many inequalities in analysis comes from using convexity. If a real function defined on an interval is convex, then the secant line which connects any two points of the given convex function lies above the graph of the given function. It can be written as

$$
f\left((1-\alpha) x_{1}+\alpha x_{2}\right) \leq(1-\alpha) f\left(x_{1}\right)+\alpha f\left(x_{2}\right)
$$

where $f$ is a convex function, any two points $x_{1}, x_{2}$ are in $\operatorname{Dom}(f)$, and $0 \leq \alpha \leq 1$.

Jensen's Inequality proved by Danish mathematician J. Jensen in 1906 that connects the value of a convex function of an integral and the integral of the convex function. One of its forms can be stated as

$$
f\left(\sum_{i=1}^{n} \alpha_{i} x_{i}\right) \leq \sum_{i=1}^{n} \alpha_{i} f\left(x_{i}\right)
$$

where $f$ is a convex function, $x_{1}, \cdots, x_{n}$ are in $\operatorname{Dom}(f)$, and $\alpha_{1}, \cdots, \alpha_{n}$ are positive weights with $\sum_{j=1}^{n} \alpha_{j}=1$ (see [3, p.90]). We note that if $f$ is a concave function, the opposite inequality holds.

If we take $f(x)=x^{k}$ for $x \geq 0$ with $k \in \mathbb{N} \cup\{0\}$ and each $\alpha_{i}=1 / n$, then

$$
\left(\frac{x_{1}+\cdots+x_{n}}{n}\right)^{k} \leq \frac{1}{n}\left(x_{1}{ }^{k}+\cdots+x_{n}{ }^{k}\right) .
$$

Received by the editors June 10, 2012. Revised July 31, 2012. Accepted August 10, 2012. 2000 Mathematics Subject Classification. 26D15.

Key words and phrases. Power series, Nesbitt's Inequality, Jensen's Inequality, Cauchy-Schwarz Inequality, arithmetic mean, geometric mean. 
For reference, see [3, p.93]. If $k=2$, it gives us the inequality between the arithmetic and quadratic means.

If we take $f(x)=e^{x}$ for real $x$ and each $\alpha_{i}=1 / n$, then

$$
e^{\frac{1}{n}\left(x_{1}+\cdots+x_{n}\right)} \leq \frac{1}{n}\left(e^{x_{1}}+\cdots+e^{x_{n}}\right) .
$$

By putting $y_{i}=e^{x_{i}}$, we get the inequality between the arithmetic and geometric means of $n$ positive numbers via

$$
\frac{y_{1}+y_{2}+\cdots+y_{n}}{n} \geq \sqrt[n]{y_{1} y_{2} \cdots y_{n}}
$$

as a special case of Jensen's inequality (see [5, p.64]).

Nesbitt's Inequality in 1903 is as follows: The inequality

$$
\frac{a}{b+c}+\frac{b}{c+a}+\frac{c}{a+b} \geq \frac{3}{2}
$$

holds for positive real numbers $a, b, c$. It is a well-known inequality with many proofs. It can be proved by comparing the arithmetic and harmonic means, or by using the arithmetic and geometric means, or by the Cauchy-Schwarz Inequality, and so on (see $[10])$.

It is generalized to the Shapiro Inequality proposed by H. Shapiro in 1954 and it is as follows: Let $x_{1}, \cdots, x_{n}$ be positive real numbers with $n \in \mathbb{N}$. If $n$ is even with $n \leq 12$, or $n$ is odd with $n \leq 23$, then

$$
\sum_{i=1}^{n} \frac{x_{i}}{x_{i+1}+x_{i+2}} \geq \frac{n}{2}
$$

where $x_{n+1}=x_{1}, x_{n+2}=x_{2}$ (see [2], [11], [12])

Now, in this paper we make several modified forms of inequalities from Nesbitt's Inequality using power series and Cauchy-Schwarz Inequality.

\section{Inequality via Power Series}

C. Mortici [4] proved Nesbitt's Inequality by using power series under the condition $a+b+c=1$ without loss of generality and it is as follows:

Let $a, b, c$ be positive real numbers with $a+b+c=1$. Then, the inequality

$$
\frac{a}{1-a}+\frac{b}{1-b}+\frac{c}{1-c} \geq \frac{3}{2}
$$

holds. Now, we modify it to get the following proposition. First of all, we note that when $0<x<1$, we have power series expansion 


$$
\frac{1}{1-x}=\sum_{k=0}^{\infty} x^{k}
$$

Proposition 2.1. Let $a, b, c$ be positive real numbers with $a+b+c=1$. Then, the inequality

$$
\frac{1}{1-a}+\frac{1}{1-b}+\frac{1}{1-c} \geq \frac{9}{2}
$$

holds.

Proof. Since $a, b, c$ are positive with $a, b, c<1$, we have power series expansions

$$
\frac{1}{1-a}+\frac{1}{1-b}+\frac{1}{1-c}=\sum_{k=0}^{\infty} a^{k}+\sum_{k=0}^{\infty} b^{k}+\sum_{k=0}^{\infty} c^{k} .
$$

These are convergent series. By applying (1.1),

$$
\sum_{k=0}^{\infty}\left(\frac{a^{k}+b^{k}+c^{k}}{3}\right) \geq \sum_{k=0}^{\infty}\left(\frac{a+b+c}{3}\right)^{k} .
$$

Therefore,

$$
\frac{1}{1-a}+\frac{1}{1-b}+\frac{1}{1-c} \geq \sum_{k=0}^{\infty} 3 \cdot\left(\frac{1}{3}\right)^{k}=3 \cdot \frac{3}{2}
$$

since $a+b+c=1$.

Corollary 2.2. Let $a, b, c$ be positive real numbers with $a+b+c=1$. Then, the inequality

$$
\frac{a^{l}}{1-a}+\frac{b^{l}}{1-b}+\frac{c^{l}}{1-c} \geq 3 \cdot \frac{3}{2} \cdot\left(\frac{1}{3}\right)^{l}
$$

holds for $l \in \mathbb{N} \cup\{0\}$.

By differentiating both sides of the equation (1.1) we get

$$
\frac{1}{(1-x)^{2}}=\sum_{k=1}^{\infty} k x^{k-1}
$$

where $0<x<1$ (see [6, p.149]). Hence, we get

Proposition 2.3. Let $a, b, c$ be positive real numbers with $a+b+c=1$. Then, the inequality

$$
\frac{1}{(1-a)^{2}}+\frac{1}{(1-b)^{2}}+\frac{1}{(1-c)^{2}} \geq 3 \cdot\left(\frac{3}{2}\right)^{2}
$$

holds. 
Proof. By power series expansion in (2.2) and the inequality in (1.1),

$$
\begin{aligned}
& \frac{1}{(1-a)^{2}}+\frac{1}{(1-b)^{2}}+\frac{1}{(1-c)^{2}} \\
= & \sum_{k=1}^{\infty} k a^{k-1}+\sum_{k=1}^{\infty} k b^{k-1}+\sum_{k=1}^{\infty} k c^{k-1} \\
= & \sum_{k=1}^{\infty} 3 k \cdot\left(\frac{a^{k-1}+b^{k-1}+c^{k-1}}{3}\right) \geq \sum_{k=1}^{\infty} 3 k \cdot\left(\frac{a+b+c}{3}\right)^{k-1} .
\end{aligned}
$$

Hence,

$$
\begin{aligned}
\frac{1}{(1-a)^{2}}+\frac{1}{(1-b)^{2}}+\frac{1}{(1-c)^{2}} & \geq \sum_{k=1}^{\infty} 3 k \cdot\left(\frac{1}{3}\right)^{k-1} \\
& =3 \cdot \frac{1}{\left(1-\frac{1}{3}\right)^{2}}=3 \cdot\left(\frac{3}{2}\right)^{2}
\end{aligned}
$$

For positive real numbers $a_{i}$ satisfying $\sum_{i=1}^{n} a_{i}=1$, we get the following.

Corollary 2.4. Let $a_{1}, \cdots, a_{n}$ be positive real numbers with $\sum_{i=1}^{n} a_{i}=1$. Then, the inequality

$$
\sum_{i=1}^{n} \frac{1}{\left(1-a_{i}\right)^{2}} \geq n \cdot\left(\frac{n}{n-1}\right)^{2}
$$

holds.

Now, we want to generalize the above inequality. By differentiating both sides of the equation (2.1) up to $m-1$ times, we get

$$
\frac{(m-1) !}{(1-x)^{m}}=\sum_{k=m-1}^{\infty} k(k-1) \cdots(k-(m-2)) x^{k-(m-1)}
$$

where $0<x<1$.

Theorem 2.5. Let $a_{1}, \cdots, a_{n}$ be positive real numbers with $\sum_{i=1}^{n} a_{i}=1$. Then, the inequality

$$
\sum_{i=1}^{n} \frac{1}{\left(1-a_{i}\right)^{m}} \geq n \cdot\left(\frac{n}{n-1}\right)^{m}
$$

holds for any $m \in \mathbb{N} \cup\{0\}$.

Proof. For any $a_{i} \in(0,1)$, we have by $(2.3)$

$$
\frac{1}{\left(1-a_{i}\right)^{m}}=\frac{1}{(m-1) !} \sum_{k=m-1}^{\infty} k(k-1) \cdots(k-(m-2)) a_{i}^{k-(m-1)} .
$$


It is a convergent series. Hence,

$$
\begin{aligned}
& \sum_{i=1}^{n} \frac{1}{\left(1-a_{i}\right)^{m}} \\
= & \sum_{i=1}^{n} \frac{1}{(m-1) !} \sum_{k=m-1}^{\infty} k(k-1) \cdots(k-(m-2)) a_{i}{ }^{k-(m-1)} \\
= & \frac{1}{(m-1) !} \sum_{k=m-1}^{\infty} k(k-1) \cdots(k-(m-2)) \sum_{i=1}^{n} a_{i}{ }^{k-(m-1)} .
\end{aligned}
$$

By using (1.1)

$$
\begin{aligned}
\sum_{i=1}^{n} a_{i}{ }^{k-(m-1)} & =n \cdot \sum_{i=1}^{n} \frac{a_{i}^{k-(m-1)}}{n} \\
& \geq n \cdot\left(\frac{\sum_{i=1}^{n} a_{i}}{n}\right)^{k-(m-1)}=n \cdot\left(\frac{1}{n}\right)^{k-(m-1)} .
\end{aligned}
$$

Therefore, we have

$$
\begin{aligned}
& \sum_{i=1}^{n} \frac{1}{\left(1-a_{i}\right)^{m}} \\
\geq & \frac{n}{(m-1) !} \sum_{k=m-1}^{\infty} k(k-1) \cdots(k-(m-2))\left(\frac{1}{n}\right)^{k-(m-1)} \\
= & \frac{n}{(m-1) !} \cdot \frac{(m-1) !}{(1-1 / n)^{m}}=n \cdot\left(\frac{n}{n-1}\right)^{m}
\end{aligned}
$$

by using power series in (2.3).

Corollary 2.6. Let $a_{1}, \cdots, a_{n}$ be positive real numbers with $\sum_{i=1}^{n} a_{i}=1$. Then, the inequality

$$
\sum_{i=1}^{n} \frac{a_{i}^{l}}{\left(1-a_{i}\right)^{m}} \geq n \cdot\left(\frac{n}{n-1}\right)^{m} \cdot\left(\frac{1}{n}\right)^{l}
$$

holds for any $l, m \in \mathbb{N} \cup\{0\}$.

We will make a modified form of the previous inequality by changing the term $1-a_{i}$ in the denominator by $1-a_{i}^{k}$. First of all, a modified form of Proposition 2.1 is as follows:

Proposition 2.7. Let $a, b, c$ be positive real numbers with $a+b+c=1$. Then, the inequality

$$
\frac{1}{1-a^{2}}+\frac{1}{1-b^{2}}+\frac{1}{1-c^{2}} \geq 3 \cdot\left(\frac{3^{2}}{3^{2}-1}\right)
$$

holds. 
If we apply the method similar to Corollary 2.6, then we get the following.

Theorem 2.8. Let $a_{1}, \cdots, a_{n}$ be positive real numbers with $\sum_{i=1}^{n} a_{i}=1$. Then, the inequality

$$
\sum_{i=1}^{n} \frac{a_{i}^{l}}{\left(1-a_{i}^{k}\right)^{m}} \geq n \cdot\left(\frac{n^{k}}{n^{k}-1}\right)^{m} \cdot\left(\frac{1}{n^{k}}\right)^{l}
$$

holds for any $l, m \in \mathbb{N} \cup\{0\}$ and $k \in \mathbb{N}$.

\section{Inequality via Cauchy-Schwarz Inequality}

The Cauchy-Schwarz Inequality is very useful in showing inequalities and finite version of it in $\mathbb{R}^{n}$ is as follows: For $x_{1}, \cdots, x_{n}, y_{1}, \cdots, y_{n} \in \mathbb{R}, n \in \mathbb{N}$,

$$
\left(\sum_{i=1}^{n} x_{i}^{2}\right) \cdot\left(\sum_{i=1}^{n} y_{i}^{2}\right) \geq\left(\sum_{i=1}^{n} x_{i} y_{i}\right)^{2} .
$$

Equality holds when $x=\left(x_{1}, \cdots, x_{n}\right)$ is a multiple of $y=\left(y_{1}, \cdots, y_{n}\right)$.

F. Zejnulahi and Š. Arlanagić [8] states that for nonnegative real numbers $a, b, c$ satisfying $a+b+c=3$, the inequalities

$$
\begin{gathered}
\frac{a^{2}}{b+1}+\frac{b^{2}}{c+1}+\frac{c^{2}}{a+1} \geq \frac{3}{2}, \\
\frac{a}{b^{2}+1}+\frac{b}{c^{2}+1}+\frac{c}{a^{2}+1} \geq \frac{3}{2}
\end{gathered}
$$

hold. Now, we modify (3.1) to get the following inequality.

Theorem 3.1. Let $a, b, c$ be nonnegative real numbers with $a+b+c=1$. Then, we have the inequality

$$
\frac{1}{1+a}+\frac{1}{1+b}+\frac{1}{1+c} \geq \frac{9}{4}
$$

and equality holds when $a=b=c=1 / 3$.

Proof. By using the Cauchy-Schwarz Inequality, we have

$$
((1+a)+(1+b)+(1+c)) \cdot\left(\frac{1}{1+a}+\frac{1}{1+b}+\frac{1}{1+c}\right) \geq(1+1+1)^{2} .
$$

Hence,

$$
4\left(\frac{1}{1+a}+\frac{1}{1+b}+\frac{1}{1+c}\right) \geq 9
$$

and it implies the desired inequality. When we use the Cauchy-Schwarz Inequality, equality holds if and only if

$$
\frac{\sqrt{1+a}}{1 / \sqrt{1+a}}=\frac{\sqrt{1+b}}{1 / \sqrt{1+b}}=\frac{\sqrt{1+c}}{1 / \sqrt{1+c}} .
$$


Hence equality in (3.3) holds when $a=b=c=1 / 3$.

On the other hand, F. Zejnulahi and Š. Arlanagić [7] asked whether for nonnegative real numbers $a, b, c$ with $a+b+c=1$, the inequality

$$
\frac{a}{b^{2}+1}+\frac{b}{c^{2}+1}+\frac{c}{a^{2}+1} \geq \frac{9}{10}
$$

holds or not. We know that equality holds if $a=b=c=1 / 3$. But, unfortunately the inequality in 3.4 was disproved in [9].

Now, it is natural to ask whether for nonnegative real numbers $a, b, c$ satisfying $a+b+c=1$, the inequality

$$
\frac{1}{1+a^{2}}+\frac{1}{1+b^{2}}+\frac{1}{1+c^{2}} \geq 3 \cdot \frac{9}{10}
$$

holds or not. As we guess, it is not true.

Example 3.2. If we take $a=1 / 3, b=2 / 3, c=0$, then

$$
\frac{1}{1+a^{2}}+\frac{1}{1+b^{2}}+\frac{1}{1+c^{2}}=337 / 130<3 \cdot \frac{9}{10} \text {. }
$$

We determine a lower bound of $1 /\left(1+a^{2}\right)+1 /\left(1+b^{2}\right)+1 /\left(1+c^{2}\right)$ in the following theorem.

Theorem 3.3. Let $a, b, c$ be nonnegative real numbers with $a+b+c=1$. Then, we have the inequality

$$
\frac{1}{1+a^{2}}+\frac{1}{1+b^{2}}+\frac{1}{1+c^{2}}>\frac{9}{4}
$$

Proof. By using the Cauchy-Schwarz Inequality, we have

$$
\left(\left(1+a^{2}\right)+\left(1+b^{2}\right)+\left(1+c^{2}\right)\right)\left(\frac{1}{1+a^{2}}+\frac{1}{1+b^{2}}+\frac{1}{1+c^{2}}\right) \geq(1+1+1)^{2} .
$$

Hence, it holds that

$$
\frac{1}{1+a^{2}}+\frac{1}{1+b^{2}}+\frac{1}{1+c^{2}} \geq \frac{9}{a^{2}+b^{2}+c^{2}+3}
$$

with equality if and only if $a=b=c=1 / 3$.

On the other hand, we note that

$$
a^{2}+b^{2}+c^{2}+3 \leq a+b+c+3=1+3=4
$$

with equality instead of inequality only when one of $a, b, c$ is 1 with other two zeros.

Hence, there are no nonnegative integers $a, b, c$ satisfying the two equalities in (3.6) and (3.7) at the same time. Hence the formula (3.5) with strict inequality holds. 
Finally, we get the minimum of $1 /\left(1+a^{2}\right)+1 /\left(1+b^{2}\right)+1 /\left(1+c^{2}\right)$ in the following theorem by modifying the proof of (3.2) in [1].

Theorem 3.4. Let $a, b, c$ be nonnegative real numbers with $a+b+c=1$. Then, we have the inequality

$$
\frac{1}{1+a^{2}}+\frac{1}{1+b^{2}}+\frac{1}{1+c^{2}} \geq \frac{5}{2}
$$

with equality when one of $a, b, c$ is 1 and the other two are zeros.

Proof. Since $a^{2}+1 \geq 2 a$, we have

$$
\frac{1}{1+a^{2}}=1-\frac{a^{2}}{a^{2}+1} \geq 1-\frac{a^{2}}{2 a}=1-\frac{a}{2} \text {. }
$$

Similaly we get

$$
\frac{1}{1+b^{2}}=1-\frac{b}{2}, \frac{1}{1+c^{2}}=1-\frac{c}{2} .
$$

Therefore, we have

$$
\frac{1}{1+a^{2}}+\frac{1}{1+b^{2}}+\frac{1}{1+c^{2}} \geq 1+1+1-\frac{a+b+c}{2}=3-\frac{1}{2}=\frac{5}{2} .
$$

Equality holds if one of $a, b, c$ is 1 and the other two are zeros.

\section{REFERENCES}

1. M. Aassila, M. Bataille \& B. Stergiou: Solution 2994. Crux Mathematicorum with Mathematical Mayhem 31 (2005), no. 8, 560-561.

2. P.J. Bushell \& J.B. MeLeod: Shapiro's Cyclic Inequality for Even $n$. J. Inequal. \& Appl. 7 (2002), no. 3, 331-349.

3. A. Lohwater: Introduction to Inequalities. e-book in PDF. http://www.mediafire.com/ ?1mw1tkgozzu, 1982.

4. C. Mortici: A Power Series Approach to Some Inequalities. Amer. Math. Monthly 119 (2012), no. 2, 147-151.

5. W. Rudin: Real \& Complex Analysis. McGraw-Hill Inc., 1974.

6. H. Silverman: Complex variables. Houghton Mifflin, 1975.

7. F. Zejnulahi \& Š. Arlanagić: Problem 2993. Crux Mathematicorum with Mathematical Mayhem 30 (2004), no. 8, 503.

8. ___ Problem 2994. Crux Mathematicorum with Mathematical Mayhem 30 (2004), no. 8,503 .

9. L. Zhou: Solution 2993. Crux Mathematicorum with Mathematical Mayhem 31 (2005), no. 8,559 .

10. http://en.wikipedia.org/wiki/Nesbitt's_inequality. 
11. http://en.wikipedia.org/wiki/Shapiro_inequality.

12. http://planetmath.org/ShapiroInequality.html.

Department of Mathematics, University of Suwon, Gyeonggi-do 445-743, Korea

Email address: mjeong@suwon.ac.kr 